\title{
Adiabatic theorems and reversible isothermal processes
}

\section{Journal Article}

Author(s):

Abou-Salem, Walid K.; Fröhlich, Jürg

Publication date:

2005-05

Permanent link:

https://doi.org/10.3929/ethz-b-000032432

Rights / license:

In Copyright - Non-Commercial Use Permitted

Originally published in:

Letters in Mathematical Physics 72(2), https://doi.org/10.1007/s11005-005-4838-1 


\title{
Adiabatic Theorems and Reversible Isothermal Processes
}

\author{
WALID K. ABOU-SALEM ${ }^{\star}$ and JÜRG FRÖHLICH \\ Institute for Theoretical Physics, ETH-Hönggerberg, CH-8093 Zürich, Switzerland. \\ e-mail: sabou@itp.phys.ethz.ch, juerg@itp.phys.ethz.ch
}

(Received: 17 January 2005; revised version: 25 March 2005)

\begin{abstract}
Isothermal processes of a finitely extended, driven quantum system in contact with an infinite heat bath are studied from the point of view of quantum statistical mechanics. Notions like heat flux, work and entropy are defined for trajectories of states close to, but distinct from states of joint thermal equilibrium. A theorem characterizing reversible isothermal processes as quasi-static processes ("isothermal theorem") is described. Corollaries concerning the changes of entropy and free energy in reversible isothermal processes and on the 0th law of thermodynamics are outlined.
\end{abstract}

Mathematics Subject Classifications (2000). 81Q05, 81Q10, 82C10.

Key words. adiabatic theorem, isothermal processes, reversible processes.

\section{Introduction}

The problem to derive the fundamental laws of thermodynamics, the 0th, 1st, 2nd (and 3rd) law, from kinetic theory and non-equilibrium statistical mechanics has been studied since the late 19th century, with contributions by many distinguished theoretical physicists including Maxwell, Boltzmann, Gibbs and Einstein. In this note, we report on some recent results concerning isothermal processes that have grown out of our own modest attempts to bring the problem just described a little closer to a satisfactory solution (see [1] for a synopsis of our results).

In the study of the 0th law and of Carnot processes isothermal processes play an important role. Such processes arise when a system with finitely many degrees of freedom, the "small system", is coupled diathermally to an infinitely extended, dispersive system, the "heat bath", e.g., one consisting of black-body radiation, or of a gas of electrons (metals), or of magnons (magnetic materials), at positive temperature. (Diathermal contacts are couplings that preserve all extensive quantities except for the "internal energy" of the "small system". The latter will be precisely defined later.) During the past ten years, a particular phenomenon, "return to equilibrium", encountered in the study of isothermal processes of quantum-mechanical systems, has been analyzed for simple (non-interacting) models of heat baths, by

\footnotetext{
^ Supported by the Swiss National Foundation.
} 
several groups of people [2-6]: After coupling the small system to the heat bath, the state of the coupled system approaches an equilibrium state at the temperature of the heat bath, as time $t$ tends to $\infty$. Further, if all contacts of the heat bath to its environment are broken the state of the heat bath returns to its original equilibrium state $[7,8]$. (The state of an isolated infinite heat bath is thus characterized by a single quantity: its temperature!)

The results in [2-6] are proven using spectral- and resonance theory, starting from the formalism developed in [9]. If $\mathcal{H}^{\mathcal{R}}$ denotes the Hilbert space of state vectors of the infinite heat bath, $\mathcal{R}$, at a fixed temperature $(k \beta)^{-1}$, where $k$ denotes Boltzmann's constant, $\left(\mathcal{H}^{\mathcal{R}}\right.$ is obtained with the help of the GNS construction, see $[9,10])$, and $\mathcal{H}^{\Sigma}$ denotes the Hilbert space of pure state vectors of the "small system" $\Sigma$, the Hilbert space of general (in particular mixed) states of the composed system, $\mathcal{R} \vee \Sigma$, is given by

$$
\mathcal{H} \equiv \mathcal{H}^{\mathcal{R} \vee \Sigma}:=\mathcal{H}^{\mathcal{R}} \otimes\left(\mathcal{H}^{\Sigma} \otimes \mathcal{H}^{\Sigma}\right)
$$

see $[4,9]$. The equilibrium state of the heat bath, or reservoir, $\mathcal{R}$ at inverse temperature $k \beta$ corresponds to a vector $\Omega_{\beta}^{\mathcal{R}} \in \mathcal{H}^{\mathcal{R}}$, and a general mixed state of the small system $\Sigma$ can be described as the square root of a density matrix, which is a Hilbert-Schmidt operator on $\mathcal{H}^{\Sigma}$, or, in other terms, as a vector in $\mathcal{H}^{\Sigma} \otimes \mathcal{H}^{\Sigma}$.

The dynamics of the composed system is generated by an (in general, timedependent) thermal Hamiltonian, or Liouvillian,

$$
L(t):=L_{0}(t)+g(t) I,
$$

(the "standard Liouvillian", see, e.g., [6,9]), where

$$
L_{0}(t)=L_{\beta}^{\mathcal{R}} \otimes(\mathbf{1} \otimes \mathbf{1})+\mathbf{1} \otimes\left(H_{0}^{\Sigma}(t) \otimes \mathbf{1}\right)-\mathbf{1} \otimes\left(\mathbf{1} \otimes H_{0}^{\Sigma}(t)\right)
$$

is the Liouvillian of the uncoupled system, $L_{\beta}^{\mathcal{R}}$ is the Liouvillian of the heat bath, with $L_{\beta}^{\mathcal{R}} \Omega_{\beta}^{\mathcal{R}}=0, H_{0}^{\Sigma}(t)$ is the (generally time-dependent) Hamiltonian of the small system, and where $g(t) I$ is a spatially localized term describing the interactions between $\mathcal{R}$ and $\Sigma$, with a time-dependent coupling constant $g(t)$. Concrete models are analyzed in [2-7].

We will only consider heat baths with a unique equilibrium state at each temperature; (no phase coexistence). If $L_{0}(t) \equiv L_{0}$ and $g(t) \equiv g$ are independent of $t$, for $t \geqslant t_{*}$, "return to equilibrium" holds true if we can prove that $L$ has a simple eigenvalue at 0 and that the spectrum, $\sigma(L)$, of $\mathrm{L}$ is purely continuous away from 0 ; see [2-4]. The eigenvector, $\Omega_{\beta} \equiv \Omega_{\beta}^{\mathcal{R} \vee \Sigma}$, of $L$ corresponding to the eigenvalue 0 is the thermal equilibrium state of the coupled system $\mathcal{R} \vee \Sigma$ at temperature $(k \beta)^{-1}$. Since $L_{0}$ tends to have a rich spectrum of eigenvalues (embedded in continuous spectrum), it is an a priori surprising consequence of interactions between $\mathcal{R}$ and $\Sigma$ that the point spectrum of $L$ consists of only one simple eigenvalue at 0 ; (see [2-6,11] for hypotheses on the interaction $I$ and results). Under suitable hypotheses on $\mathcal{R}$ and $\Sigma$, see [2-4], one can actually prove that "return to equilibrium" is described by an exponential law involving a finite relaxation time, $\tau_{\mathcal{R}}$. 
If the Liouvillian $L(t)$ of $\mathcal{R} \vee \Sigma$ depends on time $t$, but with the property that, for all times $t, L(t)$ has a simple eigenvalue at 0 corresponding to an eigenvector $\Omega_{\beta}(t)$, then $\Omega_{\beta}(t)$ can be viewed as an instantaneous equilibrium (or reference) state, and $\tau_{\mathcal{R}}(t)$ is called instantaneous relaxation time of $\mathcal{R} \vee \Sigma$. Let $\tau$ be the time scale over which $L(t)$ changes appreciably. Assuming that, at some time $t_{0}$, the state, $\Psi\left(t_{0}\right)$, of $\mathcal{R} \vee \Sigma$ is given by $\Omega_{\beta}\left(t_{0}\right)$, it is natural to compare the state $\Psi(t)$ of $\mathcal{R} \vee \Sigma$ at a later time $t$ with the instantaneous equilibrium state $\Omega_{\beta}(t)$ and to estimate the norm of the difference $\Psi(t)-\Omega_{\beta}(t)$. One would expect that if $\tau \gg \sup _{t} \tau_{\mathcal{R}}(t)$, then

$$
\Psi(t) \simeq \Omega_{\beta}(t) .
$$

In [12], we prove an "adiabatic theorem", which we call "isothermal theorem", saying that

$$
\Psi(t) \stackrel{\tau \rightarrow \infty}{\rightarrow} \Omega_{\beta}(t),
$$

for all times $t \geqslant t_{0}$. The purpose of our note is to carefully state this theorem and various generalizations thereof and to explain some of its consequences; e.g., to show that quasi-static $(\tau \rightarrow \infty)$ isothermal processes are reversible and that, in the quasi-static limit, a variant of the 0th law holds. We also propose general definitions of heat flux and of entropy for trajectories of states of $\mathcal{R} \vee \Sigma$ sampled in arbitrary isothermal processes and use the "isothermal theorem" to relate these definitions to more common ones. Details will appear in [1,12].

\section{A General "Adiabatic Theorem"}

In this section we carefully state a general adiabatic theorem which is a slight improvement of results in $[13,14]$ concerning adiabatic theorems for Hamiltonians without spectral gaps. Our simplest result follows from those in $[13,14]$ merely by eliminating the superfluous hypothesis of semiboundedness of the generator of time evolution.

Let $\mathcal{H}$ be a separable Hilbert space, and let $\{L(s)\}_{s \in I}$, with $I \subset \mathbb{R}$ a compact interval, be a family of selfadjoint operators on $\mathcal{H}$ with the following properties:

(A1) The operators $L(s), s \in I$, are selfadjoint on a common domain, $\mathcal{D}$, of definition dense in $\mathcal{H}$.

(A2) The resolvent $R(i, s):=(L(s)-i)^{-1}$ is bounded and differentiable, and $L(s) \dot{R}(i, s)$ is bounded uniformly in $s \in I$, where $(\cdot)$ denotes the derivative with respect to $s$.

Existence of time evolution. If assumptions (A1) and (A2) hold then there exist unitary operators $\left\{U\left(s, s^{\prime}\right) \mid s, s^{\prime} \in I\right\}$ with the properties:

For all $s, s^{\prime}, s^{\prime \prime}$ in $I$,

$$
U(s, s)=\mathbf{1}, U\left(s, s^{\prime}\right) U\left(s^{\prime}, s^{\prime \prime}\right)=U\left(s, s^{\prime \prime}\right),
$$


$U\left(s, s^{\prime}\right)$ is strongly continuous in $s$ and $s^{\prime}$, and

$$
i \frac{\partial}{\partial s} U\left(s, s^{\prime}\right) \Psi=L(s) U\left(s, s^{\prime}\right) \Psi,
$$

for arbitrary $\Psi \in \mathcal{D}, s, s^{\prime}$ in $I$; ( $U$ is called a "propagator").

This result follows from, e.g., Theorems X.47a and X.70 in [15] in a straightforward way; see also Theorem 2 of Chapter XIV in [16]. (Some sufficient conditions for (A1) and (A2) to hold are discussed in [12].)

In order to prove an adiabatic theorem, one must require some additional assumptions on the operators $L(s)$.

(A3) We assume that $L(s)$ has an eigenvalue $\lambda(s)$, that $\{P(s)\}$ is a family of finite rank projections such that $L(s) P(s)=\lambda(s) P(s), P(s)$ is twice continuously differentiable in $s$ with bounded first and second derivatives, for all $s \in I$, and that $P(s)$ is the spectral projection of $L(s)$ corresponding to the eigenvalue $\lambda(s)$ for almost all $s \in I$.

We consider a quantum system whose time evolution is generated by a family of operators

$$
L_{\tau}(t):=L\left(\frac{t}{\tau}\right), \frac{t}{\tau}=: s \in I,
$$

where $\{L(s)\}_{s \in I}$ satisfies assumptions (A1)-(A3). The propagator of the system is denoted by $U_{\tau}\left(t, t^{\prime}\right)$. We define

$$
U^{(\tau)}\left(s, s^{\prime}\right):=U_{\tau}\left(\tau s, \tau s^{\prime}\right)
$$

and note that $U^{(\tau)}\left(s, s^{\prime}\right)$ solves the equation

$$
i \frac{\partial}{\partial s} U^{(\tau)}\left(s, s^{\prime}\right) \Psi=\tau L(s) U^{(\tau)}\left(s, s^{\prime}\right) \Psi, \Psi \in \mathcal{D} .
$$

Next, we define

$$
L_{a}(s):=L(s)+\frac{i}{\tau}[\dot{P}(s), P(s)]
$$

and the corresponding propagator, $U_{\mathrm{a}}^{(\tau)}\left(s, s^{\prime}\right)$, which solves the equation

$$
i \frac{\partial}{\partial s} U_{\mathrm{a}}^{(\tau)}\left(s, s^{\prime}\right) \Psi=\tau L_{\mathrm{a}}(s) U_{\mathrm{a}}^{(\tau)}\left(s, s^{\prime}\right) \Psi, \Psi \in \mathcal{D} .
$$

The propagator $U_{\mathrm{a}}^{(\tau)}$ describes what one calls the adiabatic time evolution. (Note that the operators $L_{\mathrm{a}}(s), s \in I$, satisfy (A1) and (A2), since, by (A3), $\frac{i}{\tau}[\dot{P}(s), P(s)]$ are bounded, selfadjoint operators with bounded derivative in $s$.)

Adiabatic Theorem. If assumptions (A1)-(A3) hold then

(i) $U_{\mathrm{a}}^{(\tau)}\left(s^{\prime}, s\right) P(s) U_{\mathrm{a}}^{(\tau)}\left(s, s^{\prime}\right)=P\left(s^{\prime}\right)$ (intertwining property), for arbitrary $s, s^{\prime}$ in $I$, and 
(ii) $\lim _{\tau \rightarrow \infty} \sup _{s, s^{\prime} \in I}\left\|U^{(\tau)}\left(s, s^{\prime}\right)-U_{\mathrm{a}}^{(\tau)}\left(s, s^{\prime}\right)\right\|=0$.

A proof of this result can be inferred from [14].

Remarks.

(1) We note that $U^{(\tau)}\left(s^{\prime}, s\right)=U^{(\tau)}\left(s, s^{\prime}\right)^{*}$.

(2) With more precise assumptions on the nature of the spectrum of $\{L(s)\}$, one can obtain information about the speed of convergence in (ii), as $\tau \rightarrow \infty$; see [12-14]. A powerful strategy to obtain such information is to make use of complex spectral deformation techniques, such as dilatation or spectral-translation analyticity.

These techniques also enable one to prove an

(3) Adiabatic Theorem for Resonances [17]. This result resembles the adiabatic theorem described above, but eigenstates of $L(s)$ are replaced by resonance states, and one must require the adiabatic time scale $\tau$ to be small as compared to the life time, $\tau_{\text {res }}(s)$, of a resonance of $L(s)$, uniformly in $s \in I$. (For shape resonances, the techniques in [18] are useful. Precise statements and proofs can be found in [17].) Similar ideas lead to an adiabatic theorem in non-equilibrium statistical mechanics [22].

\section{The "Isothermal Theorem"}

In this section, we turn to the study of isothermal processes of "small" driven quantum systems, $\Sigma$, in diathermal contact with a heat bath, $\mathcal{R}$, at a fixed temperature $(k \beta)^{-1}$. Our notations are as in Section 1; see, Equations (1)-(3).

Let $L_{\tau}(t):=L\left(\frac{t}{\tau}\right)$ denote the Liouvillian of the coupled system $\mathcal{R} \vee \Sigma$, where $\{L(s)\}_{s \in I}$ is as in Equations (2) and (3) of Section 1 and satisfies assumptions (A1) and (A2) of Section 2. The interval

$$
I_{\tau}=\left\{t \mid \frac{t}{\tau} \in I \subset \mathbb{R}\right\}
$$

is the time interval during which an isothermal process of $\mathcal{R} \vee \Sigma$ is studied.

We assume that $\Sigma$ is driven "slowly", i.e., that $\tau$ is large as compared to the relaxation time $\tau_{\mathcal{R}}=\max _{s \in I} \tau_{\mathcal{R}}(s)$ of $\mathcal{R} \vee \Sigma$.

Assumption (A3) of Section 2 is supplemented with the following more specific assumption.

(A4) For all $s \in I \equiv\left[s_{0}, s_{1}\right]$, the operator $L(s)$ has a single, simple eigenvalue $\lambda(s)=0$, the spectrum, $\sigma(L(s)) \backslash\{0\}$, of $L(s)$ being purely continuous away from 0 . It is also assumed that, for $s \leqslant s_{0}, L(s) \equiv L$ is independent of $s$ and has spectral properties sufficient to prove return to equilibrium $[3,4]$.

Let $\Omega_{\beta}(s) \in \mathcal{H}$ denote the eigenvector of $L(s)$ corresponding to the eigenvalue 0 , for $s \leqslant s_{1}$. Then $\Omega_{\beta}\left(\frac{t}{\tau}\right)$ is the instantaneous equilibrium state of $\mathcal{R} \vee \Sigma$ at time $t$. 
Let $P(s)=\left|\Omega_{\beta}(s)\right\rangle\left\langle\Omega_{\beta}(s)\right|$ denote the orthogonal projection onto $\Omega_{\beta}(s) ; P(s)$ is assumed to satisfy (A3), Section 2 .

Let $\Psi(t)$ be the "true" state of $\mathcal{R} \vee \Sigma$ at time $t$; in particular

$$
\Psi(t)=U_{\tau}\left(t, t^{\prime}\right) \Psi\left(t^{\prime}\right),
$$

where $U_{\tau}\left(t, t^{\prime}\right)$ is the propagator corresponding to $\left\{L_{\tau}(t)\right\}$; see Equations (5)-(7), Section 2. By the property of return to equilibrium and assumption (A4),

$$
\Psi(t)=\Omega_{\beta}, \quad t \leqslant \tau s_{0},
$$

for an arbitrary initial condition $\Psi(-\infty) \in \mathcal{H}$ at $t=-\infty$.

We set

$$
\Psi^{(\tau)}(s)=\Psi(\tau s),
$$

and note that, with the notations of Equations (7), (8), Section 2, and thanks to (11)

$$
\Psi^{(\tau)}(s)=U^{(\tau)}\left(s, s_{0}\right) \Omega_{\beta},
$$

for $s \in I$.

Isothermal Theorem. Suppose that $L(s)$ and $P(s)$ satisfy assumptions (A1)-(A3), Section 2, and (A4) above. Then

$$
\lim _{\tau \rightarrow \infty} \sup _{s \in I}\left\|\Psi^{(\tau)}(s)-\Omega_{\beta}(s)\right\|=0 .
$$

\section{Remarks.}

(1) The isothermal theorem follows readily from the adiabatic theorem of Section 2 and from Equation (13) and the definition of $\Omega_{\beta}(s)$.

(2) We define the expectation values (states)

$$
\omega_{t}^{\beta}(a):=\left\langle\Omega_{\beta}\left(\frac{t}{\tau}\right), a \Omega_{\beta}\left(\frac{t}{\tau}\right)\right\rangle
$$

and

$$
\rho_{t}(a):=\langle\Psi(t), a \Psi(t)\rangle,
$$

where $a$ is an arbitrary bounded operator on $\mathcal{H}=\mathcal{H}^{\mathcal{R} \vee \Sigma}$. Then the isothermal theorem says that

$$
\rho_{t}(a)=\omega_{t}^{\beta}(a)+\epsilon_{t}^{(\tau)}(a)
$$

where

$$
\lim _{\tau \rightarrow \infty} \frac{\left|\epsilon_{t}^{(\tau)}(a)\right|}{\| a||}=0
$$

for all times $t \in I_{\tau}$. 
(3) If the complex spectral deformation techniques of [2,3] are applicable to the analysis of the coupled system $\mathcal{R} \vee \Sigma$ then

$$
\left|\epsilon_{t}^{(\tau)}(a)\right| \leqslant \mathcal{O}\left(\tau^{-\frac{1}{2}}\right)\|a\|
$$

see $[11,17]$.

(4) All our assumptions, (A1)-(A4), can be verified for the classes of systems studied in [2-6] for which return to equilibrium has been established therein. They can also be verified for a "quantum dot" coupled to non-interacting electrons in a metal, or for an impurity spin coupled to a reservoir of non-interacting magnons; see $[1,11,12]$.

\section{4. (Reversible) Isothermal Processes}

In this section, we study general isothermal processes and use the isothermal theorem to characterize reversible isothermal processes.

It will be convenient to view the heat bath $\mathcal{R}$ as the thermodynamic limit of an increasing family of quantum systems confined to compact subsets of physical space, as discussed in $[10,19]$. The pure states of a quantum mechanical system confined to a bounded region of physical space are unit rays in a separable Hilbert space, while its mixed states are described by density matrices, which are positive trace-class operators with unit trace.

Before passing to the thermodynamic limit of the heat bath, the dynamics of the coupled system, $\mathcal{R} \vee \Sigma$, is generated by a family of time-dependent Hamiltonians

$$
H(t) \equiv H^{\mathcal{R} \vee \Sigma}(t):=H^{\mathcal{R}}+H^{\Sigma}(t),
$$

where

$$
H^{\Sigma}(t)=H_{0}^{\Sigma}(t)+g(t) W
$$

$H_{0}^{\Sigma}(t)$ is as in Section 1 , and $W$ is the interaction Hamiltonian (as opposed to the interaction Liouvillian, $I=a d_{W}$, introduced in Section 1).

Let $\mathrm{P}(t)$ denote the density matrix describing the state of the coupled system, $\mathcal{R} \vee \Sigma$, at time $t$, (before the thermodynamic limit for $\mathcal{R}$ is taken). Then $\mathrm{P}(t)$ satisfies the Liouville equation

$$
\dot{\mathrm{P}}(t)=-i[H(t), \mathrm{P}(t)]
$$

The instantaneous equilibrium, or reference state of the coupled system is given, in the canonical ensemble, by the density matrix

$$
\mathrm{P}^{\beta}(t)=Z^{\beta}(t)^{-1} \mathrm{e}^{-\beta H(t)},
$$

where

$$
Z^{\beta}(t)=\operatorname{tr}\left(\mathrm{e}^{-\beta H(t)}\right)
$$


is the partition function, and tr denotes the trace. We assume that the thermodynamic limits

$$
\begin{aligned}
& \rho_{t}(\cdot)=T D \lim _{\mathcal{R}} \operatorname{tr}(\mathrm{P}(t)(\cdot)) \\
& \omega_{t}^{\beta}(\cdot)=T D \lim _{\mathcal{R}} \operatorname{tr}\left(\mathrm{P}^{\beta}(t)(\cdot)\right)
\end{aligned}
$$

exist on a suitable kinematical algebra of operators describing $\mathcal{R} \vee \Sigma$; see $[4,10,19]$.

The equilibrium state and partition function of a finitely extended heat bath are given by

$$
\begin{aligned}
& \mathrm{P}_{\mathcal{R}}^{\beta}=\left(Z_{\mathcal{R}}^{\beta}\right)^{-1} \mathrm{e}^{-\beta H^{\mathcal{R}}}, \\
& Z_{\mathcal{R}}^{\beta}=\operatorname{tr}\left(\mathrm{e}^{-\beta H^{\mathcal{R}}}\right),
\end{aligned}
$$

respectively.

Next, we introduce thermodynamic potentials for the small system $\Sigma$ : The internal energy of $\Sigma$ in the "true" state, $\rho_{t}$, of $\mathcal{R} \vee \Sigma$ at time $t$ is defined by

$$
U^{\Sigma}(t):=\rho_{t}\left(H^{\Sigma}(t)\right)
$$

and the entropy of $\Sigma$ in the state $\rho_{t}$ at time $t$ by

$$
S^{\Sigma}(t):=-k T D \lim _{\mathcal{R}} \operatorname{tr}\left(\mathrm{P}(t)\left[\ln \mathrm{P}(t)-\ln \mathrm{P}_{\mathcal{R}}^{\beta}\right]\right) .
$$

Note that we here define $S^{\Sigma}(t)$ as a relative entropy (with the aim of subtracting the divergent contribution of the heat bath to the total entropy). A general inequality for traces, see [10], says that

$$
S^{\Sigma}(t) \leqslant 0
$$

The free energy of $\Sigma$ in an instantaneous equilibrium state, $\omega_{t}^{\beta}$, of $\mathcal{R} \vee \Sigma$ is defined by

$$
F^{\Sigma}(t):=-k T T D \lim _{\mathcal{R}} \ln \left(\frac{Z^{\beta}(t)}{Z_{\mathcal{R}}^{\beta}}\right) .
$$

Next, we define quantities associated not with states but with the thermodynamic process carried out by $\mathcal{R} \vee \Sigma$ : the heat flux into $\Sigma$ and the work rate, or power, of $\Sigma$. Let $\delta$ denote the so called "imperfect differential". Then

$$
\frac{\delta Q^{\Sigma}}{\mathrm{d} t}(t):=T D \lim _{\mathcal{R}}-\frac{\mathrm{d}}{\mathrm{d} t} \operatorname{tr}\left(\mathrm{P}(t) H^{\mathcal{R}}\right),
$$

and

$$
\frac{\delta A^{\Sigma}}{\mathrm{d} t}(t):=\rho_{t}\left(\dot{H}^{\Sigma}(t)\right)
$$

see $[1,8,12]$ for details. 
We are now prepared to summarize our main results on isothermal processes. The first two results are general and concern the first law of thermodynamics and the relationship between the rate of change of entropy and the heat flux into $\Sigma$. The remaining three results are corollaries pertaining to free energy and changes of entropy in reversible isothermal processes, i.e., processes in which states are sampled at equilibrium, and on the zeroth law of thermodynamics.

(1) From definitions (28), (32) and (33) and the Liouville equation (21) it follows that

$$
\dot{U}^{\Sigma}(t)=\frac{\delta Q^{\Sigma}}{\mathrm{d} t}(t)+\frac{\delta A^{\Sigma}}{\mathrm{d} t}(t)
$$

which is the first law of thermodynamics; (hardly more than a definition of $\frac{\delta A^{\Sigma}}{\mathrm{dt}}$ ).

(2) Note that, by the unitarity of time evolution and the cyclic invariance of the trace,

$$
\frac{\mathrm{d}}{\mathrm{d} t} \operatorname{tr}(\mathrm{P}(t) \ln \mathrm{P}(t))=0
$$

and

$$
\frac{\mathrm{d}}{\mathrm{d} t} \operatorname{tr}\left(\mathrm{P}(t) \ln Z_{\mathcal{R}}^{\beta}\right)=\frac{\mathrm{d}}{\mathrm{d} t} \ln Z_{\mathcal{R}}^{\beta}=0 .
$$

Together with definitions (26), (29) and (32), this implies that

$$
\dot{S}^{\Sigma}(t)=\frac{1}{T} \frac{\delta Q^{\Sigma}}{\mathrm{d} t}(t)
$$

for arbitrary isothermal processes at temperature $T=(k \beta)^{-1}$.

(3) Next, we consider an isothermal process of $\mathcal{R} \vee \Sigma$ during a finite time interval $I_{\tau}=\left[\tau s_{0}, \tau s_{1}\right]$, with $s_{0}$ and $s_{1}$ fixed. The initial state $\rho_{\tau s_{0}}$ of $\mathcal{R} \vee \Sigma$ is assumed to be an equilibrium state $\omega_{\tau s_{0}}^{\beta}$ of the Liouvillian $L_{\tau}\left(\tau s_{0}\right)=L\left(s_{0}\right)$. We are interested in the properties of such a process when $\tau$ becomes large, i.e., when the process is quasi-static.

Result. Quasi-static isothermal processes are reversible (in the sense that all intermediate states $\rho_{t}$ of $\mathcal{R} \vee \Sigma, t \in I_{\tau}$, converge in norm to instantaneous equilibrium states $\omega_{t}^{\beta}$, as $\tau \rightarrow \infty$ ).

This result is an immediate consequence of the isothermal theorem. It means that, for all practical purposes, an isothermal process with time scale $\tau$ is reversible if $\tau \gg \tau_{\mathcal{R}}=\max _{s \in I} \tau_{\mathcal{R}}(s)$.

(4) For reversible isothermal processes, the usual equilibrium definitions of internal energy and entropy of the small system $\Sigma$ can be used:

$$
\begin{aligned}
& U_{\text {rev }}^{\Sigma}(t):=\omega_{t}^{\beta}\left(H^{\Sigma}(t)\right), \\
& S_{\text {rev }}^{\Sigma}(t):=-k T D \lim _{\mathcal{R}} \operatorname{tr}\left(\mathrm{P}^{\beta}(t)\left[\ln \mathrm{P}^{\beta}(t)-\ln \mathrm{P}^{\mathcal{R}}\right]\right)=\frac{1}{T}\left(U_{\text {rev }}^{\Sigma}(t)-F^{\Sigma}(t)\right),
\end{aligned}
$$


where the free energy $F^{\Sigma}(t)$ has been defined in (31), and the second equation in (37) follows from (22), (26), (31) and (36). Eqs. (37) and (31) then imply that

$$
\dot{S}_{\mathrm{rev}}^{\Sigma}(t)=\frac{1}{T}\left(\frac{\mathrm{d}}{\mathrm{d} t} \omega_{t}^{\beta}\left(H^{\Sigma}(t)\right)-\omega_{t}^{\beta}\left(\dot{H}^{\Sigma}(t)\right)\right.
$$

Recalling (34) and (35), and applying the isothermal theorem, we find that

$$
\begin{aligned}
& \dot{S}^{\Sigma}(t) \rightarrow \dot{S}_{\mathrm{rev}}^{\Sigma}(t), \\
& \frac{\delta A^{\Sigma}}{\mathrm{d} t}(t) \rightarrow \dot{F}^{\Sigma}(t),
\end{aligned}
$$

as $\tau \rightarrow \infty$.

(5) We conclude this overview by considering a quasi-static isothermal process of $\mathcal{R} \vee \Sigma$ with $H^{\Sigma}(s) \rightarrow H_{0}^{\Sigma}, g(s) \rightarrow 0$, as $s \nearrow s_{1}$, i.e., the interactions between $\mathcal{R}$ and $\Sigma$ are switched off at the end of the process. Then the isothermal theorem implies that

$$
\lim _{\tau \rightarrow \infty} \lim _{s \nearrow s_{1}} \rho_{\tau s}=\omega_{\mathcal{R}}^{\beta} \otimes \omega_{\Sigma}^{\beta}
$$

where $\omega_{\mathcal{R}}^{\beta}(\cdot)=\left(Z_{\mathcal{R}}^{\beta}\right)^{-1} \operatorname{tr}\left(\mathrm{e}^{-\beta H^{\mathcal{R}}} \cdot\right)$, see (26), and

$$
\omega_{\Sigma}^{\beta}(\cdot)=\left(Z_{\Sigma}^{\beta}\right)^{-1} \operatorname{tr}\left(\mathrm{e}^{-\beta H_{0}^{\Sigma}} \cdot\right)
$$

is the Gibbs state of the small system $\Sigma$ at the temperature $(k \beta)^{-1}$ of the heat bath, independently of the properties of the diathermal contact (i.e., of the interaction Hamiltonian $W$ ), assuming that (A1)-(A4) hold for $s<s_{1}$.

This result and the property of return to equilibrium for the heat bath $\mathcal{R}$ yield, in essence, the $0 t h$ law of thermodynamics.

Carnot processes and the 2nd law of thermodynamics are discussed in [1]; (see also $[10,20,21])$. An important variant of the adiabatic theorem for non-equilibrium stationary states will appear in [22]. The analysis in [22] is based on some basic techniques developed in [23].

\section{References}

1. Abou-Salem, W. K. and Fröhlich, J.: Status of the fundamental laws of thermodynamics, in preparation.

2. Jaksić, V. and Pillet, C.-A.: On a model of quantum friction II: Fermi's golden rule and dynamics at positive temperature, Comm. Math. Phys. 176 (1996), 619-644.

3. Jaksić, V. and Pillet, C.-A.: On a model of quantum friction III: Ergodic properties of the spin-boson system, Comm. Math. Phys. 178 (1996), 627-651.

4. Bach, V., Fröhlich, J. and Sigal, I. M.: Return to equilibrium, J. Math. Phys. 41 (2000), 3985-4060.

5. Merkli, M.: Positive commutators in non-equilibrium quantum statistical mechanics, Comm. Math. Phys. 223 (2001), 327-362.

6. Fröhlich, J. and Merkli, M.: Another return of "return to equilibrium", Comm. Math. Phys. 251 (2004), 235-262. 
7. Robinson, D. W.: Return to equilibrium, Comm. Math. Phys. 31 (1973), 171-189.

8. Fröhlich, J., Merkli, M., Ueltschi, D. and Schwarz, S.: Statistical mechanics of thermodynamic processes, in: A garden of quanta, World Sci. Publishing, River Edge, New Jersey, 2003, 345-363.

9. Haag, R., Hugenholtz, N. M. and Winnink, M.: On equilibrium states in quantum statistical mechanics, Comm. Math. Phys. 5 (1967), 215-236.

10. Bratelli, O. and Robinson, D. W.: Operator Algebras and Quantum Statistical Mechanics I, II, Texts and Monographs in Physics, Springer-Verlag, Berlin, 1987.

11. Abou-Salem, W. K.: PhD thesis (2005).

12. Abou-Salem, W. K. and Fröhlich, J., in preparation.

13. Avron, J. E. and Elgart, A.: Adiabatic theorem without a gap condition, Comm. Math. Phys. 203 (1999), 445-463.

14. Teufel, S.: A note on the adiabatic theorem, Lett. Math. Phys. 58 (2001), 261-266.

15. Reed, M. and Simon, B.: Methods of Modern Mathematical Physics, vol. II, Academic Press, New York, 1975.

16. Yosida, K.: Functional Analysis, 6th edn., Springer-Verlag, Berlin, 1998.

17. Abou-Salem, W. K. and Fröhlich, J.: Adiabatic theorem for resonances, in preparation.

18. Fröhlich, J. and Pfeifer, P.: Generalized time-energy uncertainty relations and bounds on lifetimes of resonances, Rev. Mod. Phys. 67 (1995), 759-779.

19. Ruelle, D.: Statistical Mechanics: Rigorous Results, World Scientific, Singapore, 1999.

20. Ruelle, D.: Entropy production in quantum spin systems, Comm. Math. Phys. 224 (2001), 3-16.

21. Fröhlich, J., Merkli, M. and Ueltschi, D.: Dissipative transport: thermal contacts and tunnelling junctions, Ann. Henri Poincaré 4 (2003), 897-945.

22. Abou-Salem, W. K.: An adiabatic theorem for non-equilibrium steady states, in preparation.

23. Jaksić, V. and Pillet, C.-A.: Non-equilibrium steady states of finite quantum systems coupled to thermal reservoirs, Comm. Math. Phys. 226 (2002), 131-162. 